\title{
A lithium-containing biomaterial promotes chondrogenic differentiation of induced pluripotent stem cells with reducing hypertrophy
}

Yaqian Hu ${ }^{1,2+}$, Lei Chen ${ }^{3 \dagger}$, Yi Gao ${ }^{2}$, Pengzhen Cheng ${ }^{2}$, Liu Yang ${ }^{2 *}$, Chengtie Wu ${ }^{3}$ and Qiang Jie ${ }^{1 *}$

\begin{abstract}
Background: Induced pluripotent stem cells (iPSCs) exhibit limitless pluripotent plasticity and proliferation capability to provide an abundant cell source for tissue regenerative medicine. Thus, inducing iPSCs toward a specific differentiation direction is an important scientific question. Traditionally, iPSCs have been induced to chondrocytes with the help of some small molecules within 21-36 days. To speed up the differentiation of iPSCs, we supposed to utilize bioactive ceramics to assist chondrogenic-induction process.

Methods: In this study, we applied ionic products $(3.125 \sim 12.5 \mathrm{mg} / \mathrm{mL})$ of the lithium-containing bioceramic $\left(\mathrm{Li}_{2} \mathrm{Ca}_{4} \mathrm{Si}_{4} \mathrm{O}_{13}, \mathrm{~L} 2 \mathrm{C} 4 \mathrm{~S} 4\right)$ and individual $\mathrm{Li}^{+}(5.78 \sim 23.73 \mathrm{mg} / \mathrm{L})$ in the direct chondrogenic differentiation of human iPSCs.

Results: Compared to pure chondrogenic medium and extracts of tricalcium phosphate (TCP), the extracts of L2C4S4 at a certain concentration range $(3.125 \sim 12.5 \mathrm{mg} / \mathrm{mL})$ significantly enhanced chondrogenic proteins Type II Collagen (COL II)/Aggrecan/ SRY-Box 9 (SOX9) synthesis and reduced hypertrophic protein type X collagen (COL X)/matrix metallopeptidase 13 (MMP13) production in iPSCs-derived chondrocytes within 14 days, suggesting that these newly generated chondrocytes exhibited favorable chondrocytes characteristics and maintained a low-hypertrophy state. Further studies demonstrated that the individual $\mathrm{Li}^{+}$ions at the concentration range of $5.78 \sim 23.73 \mathrm{mg} / \mathrm{L}$ also accelerated the chondrogenic differentiation of iPSCs, indicating that $\mathrm{Li}^{+}$ions played a pivotal role in chondrogenic differentiation process.
\end{abstract}

Conclusions: These findings indicated that lithium-containing bioceramic with bioactive specific ionic components may be used for a promising platform for inducing iPSCS toward chondrogenic differentiation and cartilage regeneration.

Keywords: $\mathrm{PSCS}_{1} \mathrm{Li}_{2} \mathrm{Ca}_{4} \mathrm{Si}_{4} \mathrm{O}_{13}$ bioceramic, Chondrocytes

\section{Background}

Articular cartilage regeneration has always been a tricky problem in the field of skeleton repair. It is almost impossible for damaged cartilage to naturally heal because of its avascularity and absence of stem cell migration [1]. Produced by chondrocytes, extracellular matrix components trap chondrocytes and prevent them from

\footnotetext{
* Correspondence: yangliu@fmmu.edu.cn; jiqiang@fmmu.edu.cn

${ }^{\dagger}$ Yaqian Hu and Lei Chen contributed equally to this work.

${ }^{2}$ Institute of Orthopedic Surgery, Xijing Hospital, Fourth Military Medical University, Xi'an 710032, People's Republic of China

'Department of Orthopedic Surgery, Honghui Hospital, College of Medicine Xi'an Jiaotong University, Xi'an 710049, People's Republic of China Full list of author information is available at the end of the article
}

migrating and repairing once the cartilage is damaged. Clinical approaches treating cartilage defects usually resort to autologous cartilage or endogenous mesenchymal stem cells (MSCs) [2]; however, these approaches suffer from a limited amount of autologous cartilage and poor chondrogenic differentiation ability of aged MSCs. Therefore, it is important to generate functional chondrocytes from an adequate source.

In recent years, iPSCs were used as potentially unlimited cells for tissue regeneration. The iPSCs were considered as an attractive cell source for cartilage repair owing to their abundance, autologous nature, and potentiality to generate adequate chondrocytes rather than

(C) The Author(s). 2020 Open Access This article is distributed under the terms of the Creative Commons Attribution 4.0 International License (http://creativecommons.org/licenses/by/4.0/), which permits unrestricted use, distribution, and 
other cell sources [3]. While there was currently no generally accepted efficient protocol for differentiation chondrocytes from iPSCs [4], producing embryoid bodies (EBs) or MSC-like cells from iPSCs before chondrocytes differentiation were reported as regular methods by previous studies [5-7]. Nevertheless, these methods were time-consuming for multiple differentiation steps and would cause adverse effects on the urgency of clinical cartilage repair. Therefore, there were a few attempts to apply biomaterials for facilitating cartilage repair in a combination of iPSCs in vivo $[2,8,9]$. However, the direct use of undifferentiated iPSCsbiomaterials composites for cartilage defects repair is not completely safe because of the adventure of teratoma formation. Thus, if iPSCs can be rapidly induced into chondrocytes by means of biomaterials in vitro before implanted in cartilage defects, it will be of great significance for the urgency and safety of clinical cartilage repair. Nevertheless, to our knowledge, it is unclear whether the biomaterial can observably promote chondrogenic differentiation of iPSCs in vitro.

In order to solve these doubts, we synthesized a new L2C4S4 bioceramics which was proven to promote in vivo repair of osteochondral defects in our previous study [10]. In view of the advantageous stimulatory effects of the ionic products from L2C4S4 on chondrogenesis in vitro, we supposed that ionic products of this bioceramics may also biologically facilitate chondrogenic differentiation of iPSCs.

In this study, serial dilutions of extracts of L2C4S4 powders $(3.125 \sim 12.5 \mathrm{mg} / \mathrm{mL})$ were applied to the directly chondrogenic induction process of iPSCs, the TCP powders were served as a control. Various chondrocytes indicators were obtained and analyzed after 14 days of chondrogenic differentiation. Compared with pure chondrocyte-inducing medium or the extracts of TCP, the extracts of L2C4S4 accelerated the chondrogenic differentiation of iPSCs and significantly prevented the hypertrophy of newly derived chondrocytes. Furthermore, when individual $\mathrm{Li}^{+}$ions at different concentrations $(5.78 \sim 23.73 \mathrm{mg} / \mathrm{L})$ were applied in the chondrogenic induction of iPSCs in the same way, it also showed similar results. From the above, L2C4S4 represented a promising biomaterial for directly chondrogenic differentiation of iPSCs within a short time, and the $\mathrm{Li}^{+}$ions in this bioceramic made an important contribution to this differentiation process.

\section{Methods}

\section{Human iPSCs culture and identification}

Human iPSCs were established with the help of Shenzhen Cell Inspire Biotechnology Company and cultured in mTeSR 1 medium at $37^{\circ} \mathrm{C}$ in a humidified $\mathrm{CO}_{2}$ incubator. The medium was changed every day. The iPSCs were passaged to Matrigel-coated polystyrene plates. Before being conduced in the experiment, the iPSCs underwent a variety of tests on cell pluripotency.

Alkaline phosphatase staining: undifferentiated iPSCs were washed with PBS and fixed with $4 \%$ paraformaldehyde for $5 \mathrm{~min}$. Staining was done using an alkaline phosphatase detection kit (Beyotime Biotechnology, People's Republic of China). Cells were washed with PBS and surveyed under the microscope.

$E B$ induction and three germ layers differentiation: For EB formation, iPSCs were added onto ultra-lowattachment plates (Corning, USA) with a concentration of 3000/20ul. After 7 days of EB formation, EBs were cultured in $\alpha$-MEM medium with $10 \%$ FBS in 6-well plate for 7 days to differentiate into three germ layer cells.

\section{The cell proliferation assay}

L2C4S4- and TCP-graded extracts in mTeSR1 medium were used to culture human iPSCs for 7 days in 6-well plates. A nuclear protein Ki67 which shows cell division was detected to evaluate the cell proliferation in different L2C4S4 and TCP extracts. If the fluorescence of a nucleus (blue) and ki67 (red) co-localized, the cell was regarded as a positive cell which is proliferating. The positive cell number of each group was statistically present as mean \pm the standard error of the mean (SEM) and $p$ values of significance is calculated by Student's $t$ test (tails $=2$, two-sample unequal variance). ${ }^{*} p<0.05$; $* p<0.01$, and ${ }^{* * * *} p<0.001$, ns is no significance with $p>$ 0.05 .

\section{Preparation of the extracts of L2C4S4 and TCP powders}

The L2C4S4 bioceramic powders were synthesized and characterized as previously reported [10]. Pure TCP powders were prepared as the control. Extracts of the L2C4S4 and TCP powders were prepared following the protocol of the International Standard Organization (ISO/EN 10993-5). Briefly, steam sterilization method was utilized to sterilize the L2C4S4 and TCP powders before soaked in serum-free chondrogenic differentiation medium (MCDM; SclenCell, USA) or mTeSR1 medium (STEMCELL Technologies, Canada) at the concentration of $200 \mathrm{mg} / \mathrm{mL}$. After incubated at $37^{\circ} \mathrm{C}$ for $24 \mathrm{~h}$, the mixtures were centrifuged, and the supernatants were collected. The original extracts were sterilized using a $0.2 \mu \mathrm{m}$ filter. Subsequently, serial dilutions of extracts $(12.5,6.25$, and $3.125 \mathrm{mg} / \mathrm{mL})$ were prepared using MCDM or mTeSR1 medium for further cell culture experiments. The ionic concentrations of $\mathrm{Ca}, \mathrm{Li}$, and $\mathrm{P}$ in the graded extracts were calculated by inductively coupled plasma atomic emission spectrometry (ICP-AES, 715-ES, Varian, USA). The medium 
without the addition of material extracts was used as a blank control.

\section{Chondrocyte spheres induction with extracts of L2C4S4 and TCP}

The iPSCs were dissociated to a single cell suspension by Cell Dissociation Buffer (Gibco, USA) and then diluted to a final concentration of $3 * 10^{5} / 20 \mathrm{ul}$. Each $20 \mu \mathrm{l}$ of the cell suspension was added to a low-adherence 24well plate and cultured in the incubator at $37^{\circ} \mathrm{C}$ for $3 \mathrm{~h}$ to form a sphere. $0.7 \mathrm{~mL} \mathrm{MCDM}$ with serial dilutions of extracts $(12.5,6.25$ and $3.125 \mathrm{mg} / \mathrm{mL})$ was added to each well slowly. Cells were cultured as non-adherent spheres for 14 days. The medium was changed every other day.

\section{Chondrocyte spheres induction with $\mathrm{LiCl}$}

Ionic concentrations in graded extracts of L2C4S4 and TCP were calculated by inductively coupled plasma atomic emission spectrometry. To mimic $\mathrm{Li}^{+}$ ions concentrations in L2C4S4 extracts, 5.78 23.73 $\mathrm{mg} / \mathrm{L} \mathrm{Li}^{+}$ions were prepared of $\mathrm{LiCl}$ (China National Pharmaceutical Group Corporation, People's Republic of China) and applied in MCDM to culture iPSCs. MCDM without any extracts was served as a control. The iPSCs were dissociated and diluted to a final concentration of $3 * 10^{5} / 20 \mathrm{ul}$. Each $20 \mu \mathrm{l}$ of the cell suspension was cultured in the incubator for $3 \mathrm{~h}$ to form a sphere. $0.7 \mathrm{~mL} \mathrm{MCDM}$ with serial dilutions of $\mathrm{Li}^{+}$ions $(5.78 \sim 23.73 \mathrm{mg} / \mathrm{L})$ was added to each well. Cells were cultured as non-adherent spheres for 14 days. The medium was changed every other day.

\section{Immunofluorescence}

For immunofluorescence, frozen sections of chondrocyte spheres were washed with PBS for three times, these cell slices were permeabilized with cold $0.2 \%$ Triton X-100 (Sigma, USA) in PBS for 5 min. A step of enzymatic antigen retrieval with $0.1 \%$ Trypsin in PBS was performed prior to block for $1 \mathrm{~h}$. After blocking, antibodies against Aggrecan (1:300; Abcam, UK), COL II (1:300; Abcam), SOX9 (1:200; Affinity), MMP13 (1:200; Affinity), COL X (1:500; Abcam), NANOG (1:300; Abcam), OCT4 (1:300; Abcam), TRA-1-60(1:300; Abcam) and Ki67 (1:300; Abcam) were added overnight. The following day, cells were washed three times with PBS and then incubated at $37^{\circ} \mathrm{C}$ for $1 \mathrm{~h}$ with Alexa 594-conjugated goat antirabbit secondary antibody (1:300; Abcam) or Alexa 488-conjugated goat anti-mouse secondary antibody (1:300; Abcam). The cell nucleus was counterstained with DAPI (Beyotime, People's Republic of China).

\section{Microscopy and statistical analysis}

Fluorescent microscopy was performed on an Olympus microscope; images were taken under $\times 40$ objective. In order to compare the differences in immunofluorescence intensity between the groups intuitively, the average fluorescence intensity of the indicated proteins in the view region was analyzed using the Image-Pro Plus 6.0 system. The quantification data were statistically present as mean \pm SEM. $p$ values of significance is calculated by Student's $t$ test (tails $=2$, two-sample unequal variance): ${ }^{*} p<0.05,{ }^{* *} p<0.01,{ }^{* * *} p<0.001$, ns is no significance with $p>0.05$.

\section{Real-time polymerase chain reaction}

To evaluate the mRNA transcript levels of chondrocytes specific genes (Col2a1, Aggrecan, Sox9, Col10a1, Mmp13, and Ihh), stem cell-specific genes (Oct4, Nanog, and Sox2), and three germ layer genes (Nestin, Map 2, Desmin, Msx1, and Sox17), chondrocyte spheres, iPSCs, and EBs were processed for total RNA extraction by using an RNAprep Micro Kit (TaKaRa, Japan) at 14 days or 7 days. The concentration of RNA was determined with an RNA analyzer (Quawell, USA). The cDNA was prepared with PrimeScript RT Master Mix (TaKaRa, Japan). RT-PCR was performed by using SYBR Green QPCR Master Mix (TaKaRa, Japan) with a Light Cycler apparatus (Bio-Rad, USA). Cycle conditions were as follows: activation of HotStarTaq DNA polymerase/inactivation of reverse transcriptase at $95^{\circ} \mathrm{C}$ for $30 \mathrm{~s}$; and 39 cycles of $95^{\circ} \mathrm{C}$ for $5 \mathrm{~s}$, and $60^{\circ} \mathrm{C}$ for $30 \mathrm{~s}$. The relative expression level of each target gene was calculated by using the $2^{-\Delta \Delta \mathrm{Ct}}$ method. All of the primers' information was provided in Table. S1. Results were repeated for three independent biological replicates. The RT-PCR data were statistically present as mean \pm SEM and $p$ values of significance were calculated by Student's $t$ test (tails $=2$, Two-sample unequal variance) in Excel: ${ }^{*} p<$ $0.05,{ }^{* *} p<0.01,{ }^{* * *} p<0.001, \mathrm{~ns}$ is no significance with $p>0.05$.

\section{Results \\ Characterization of iPSCs generated by cellular reprogramming}

Human iPSCs were generated by cellular reprogramming and cultured in mTeSR 1 medium at $37^{\circ} \mathrm{C}$ in a humidified $\mathrm{CO}_{2}$ incubator. With several passages after cell thawing, iPSCs showed embryonic stem cell-like morphology during an expansion (Fig. 1a) and exhibited positive alkaline phosphatase staining (Fig. 1b). The iPSCs also displayed obvious immunofluorescence of pluripotent proteins OCT4, NANOG, and TRA-1-60 in their nucleus and cytoplasm (Fig. 1e). After culturing on ultra-low-attachment plates with a concentration of $3000 / 20 \mu \mathrm{l}$, the iPSCs formed a large number of EBs with smooth edges and uniform sizes (Fig. 1c), and these 

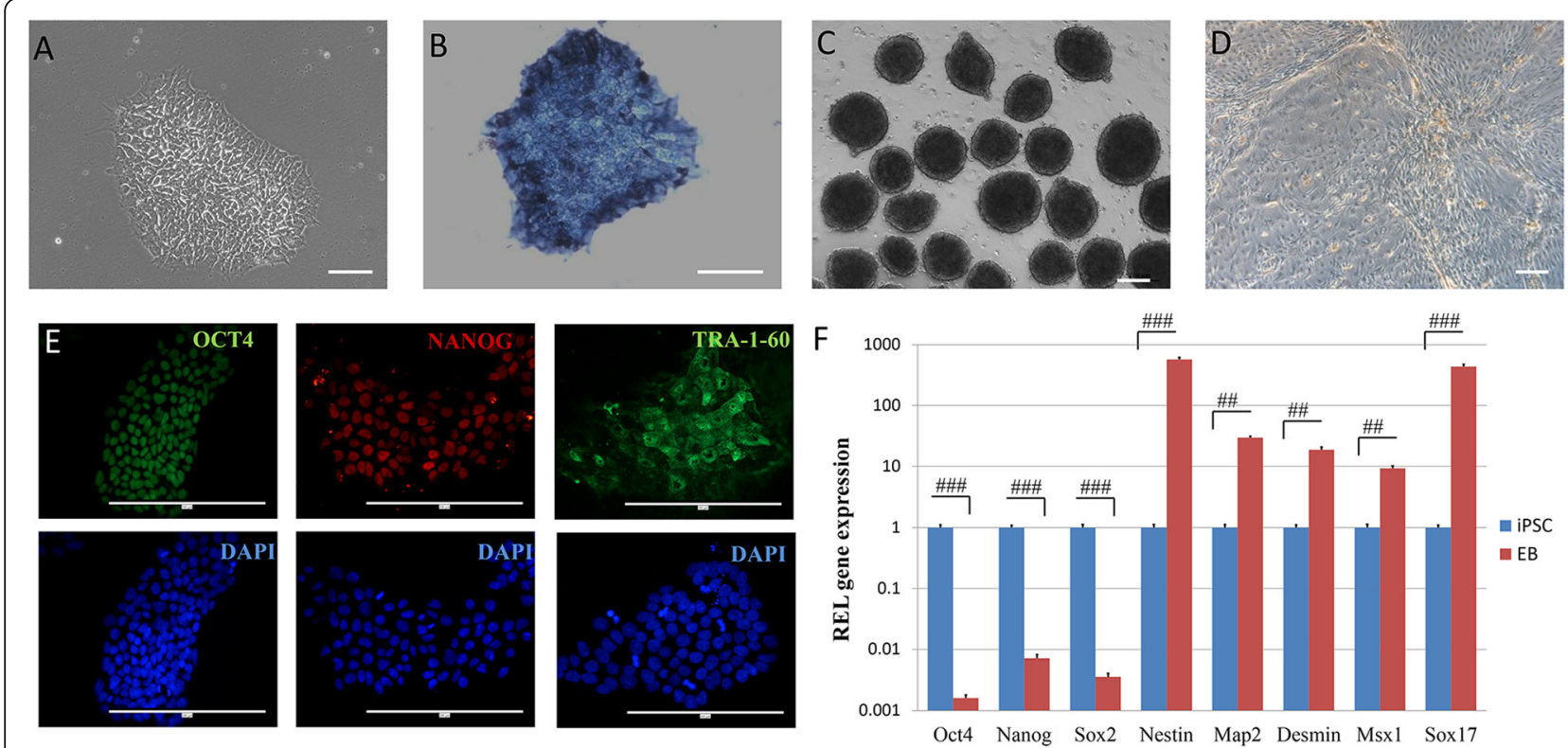

Fig. 1 The iPSCs identification. The iPSCs showed embryonic stem cell-like morphology during expansion (a) and exhibited positive alkaline phosphatase staining (b). EBs were formed (C) and able to differentiate into three germ layer cells (d). The iPSCs displayed obvious immunofluorescence of pluripotent proteins OCT4, NANOG, and TRA-1-60 (e). The expression of pluripotent genes (Oct4, Nanog, Sox2) and three germ layers genes (Nestin, Map 2, Desmin, Msx1, Sox17) were assessed in iPSCs and EBs (f). Scale bar, a and b $100 \mu$ m; c-e $200 \mu \mathrm{m}$. Data presented as mean \pm SEM. ${ }^{\# \#} p<0.01 ;{ }^{\# \# \#} p<0.001$

EBs could generate cells of three germ layers after cultured in 6-well plate for 7 days (Fig. 1d). The expression of pluripotency genes (Oct4, Nanog, Sox2), ectoderm genes (Nestin, Map 2), mesoderm genes (Desmin, Msx1), and endoderm gene (Sox17) were also assessed by RT-PCR in iPSCs and EBs. The expression level of each gene in iPSCs group was set as 1. Compared to iPSCs, the expression of pluripotency genes had obviously declined $(p<0.001)$ while the genes of the three germ layers had significantly risen $(p<0.01)$ in EBs (Fig. 1f), which indicated great potential for multidirectional differentiation of the cells. Together, these data suggested that the iPSCs exhibited the genuine characteristic of pluripotency.

\section{L2C4S4 helps keep the proliferation and pluripotency of iPSCs}

Before implementing chondrogenic differentiation with extracts of L2C4S4, we explored their effects on iPSCs proliferation to determine whether they were harmful to iPSCs. Serial dilutions of extracts (12.5, 6.25 , and $3.125 \mathrm{mg} / \mathrm{mL}$ ) of L2C4S4 and TCP were prepared with mTeSR1 medium. The iPSCs were cultured in this medium for 7 days and then underwent proliferation detection. Through fluorescence detection of proliferation marker Ki67, we found that almost every nucleus was labeled in L2C4S4-treated cells at an altered concentration $(3.125 \sim 12.5 \mathrm{mg} / \mathrm{mL})$, suggesting that all the cells were proliferating. The control group and TCP-treated cells also showed similar proliferation (Fig. 2a). In order to statistically compare the differences between the groups, we defined these ki67-stained cells as positive cells and counted the percentage of positive cells in each group. There was no statistical difference between the groups $(p>0.05$, Fig. 2b). The iPSCs maintained strong growth momentum after cultured in different concentrations of L2C4S4 extract. This revealed a strong affinity of L2C4S4 without any toxicity to the iPSCs and was important for the subsequent differentiation process.

In addition, in order to figure out whether the L2C4S4 can promote spontaneous differentiation of iPSCs, we also carried out pluripotency analyses under the same culture condition. Both of pluripotency proteins OCT4 and NANOG showed high fluorescence level in the nucleus in each group of L2C4S4 and TCP (Fig. 3a). The average fluorescence intensity of these two proteins was analyzed by the Image-Pro Plus 6.0 system. There was no statistical difference between the groups $(p>0.05$, Fig. $3 \mathrm{~b})$. In addition, to explore the effects of L2C4S4 on iPSCs' pluripotency, RT-PCR analysis of pluripotency genes (Oct4, Nanog, and Sox2) was conducted. There was also no statistical difference between each group $(p>0.05$, Fig. 3c). These results indicated that the addition of L2C4S4 extracts to the 

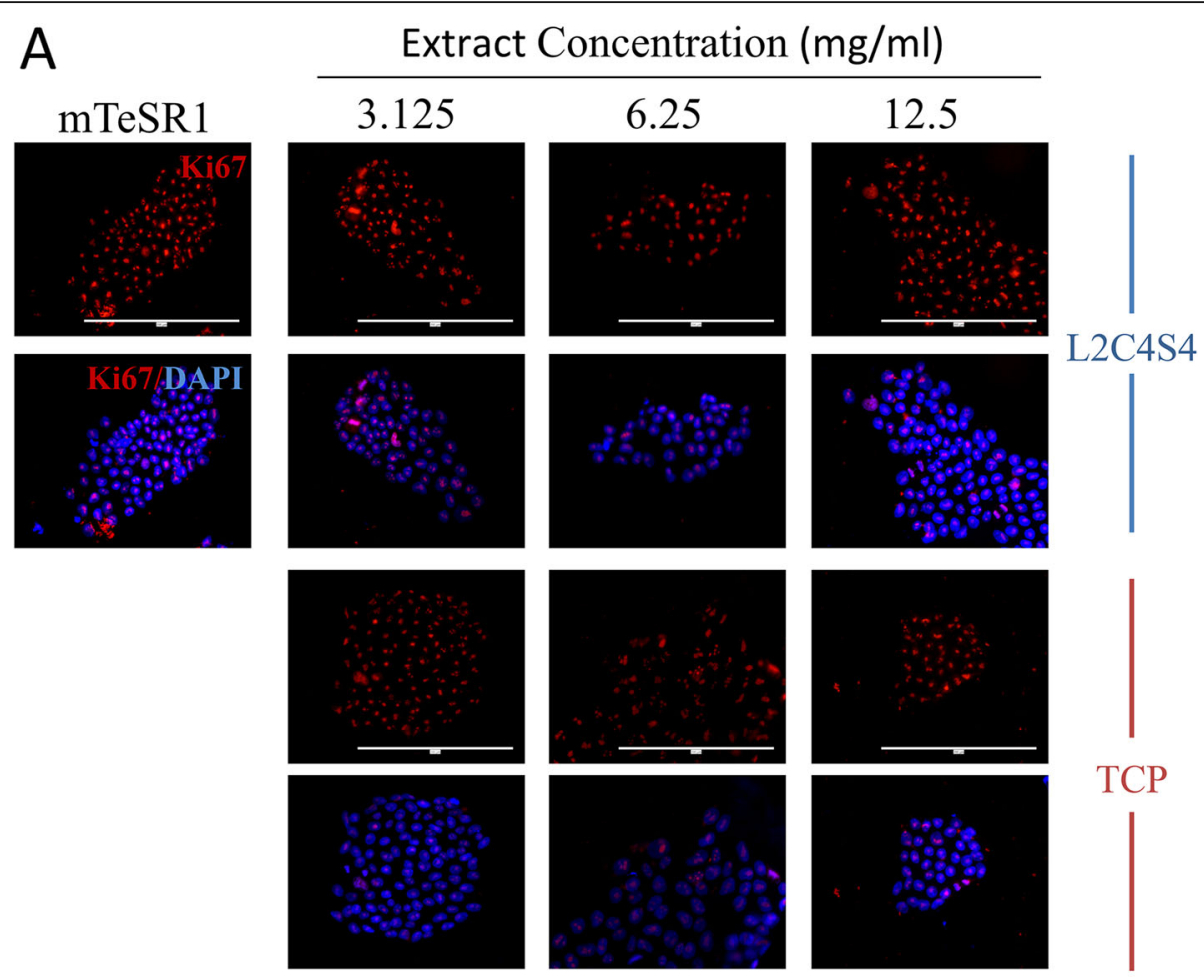

\section{B}

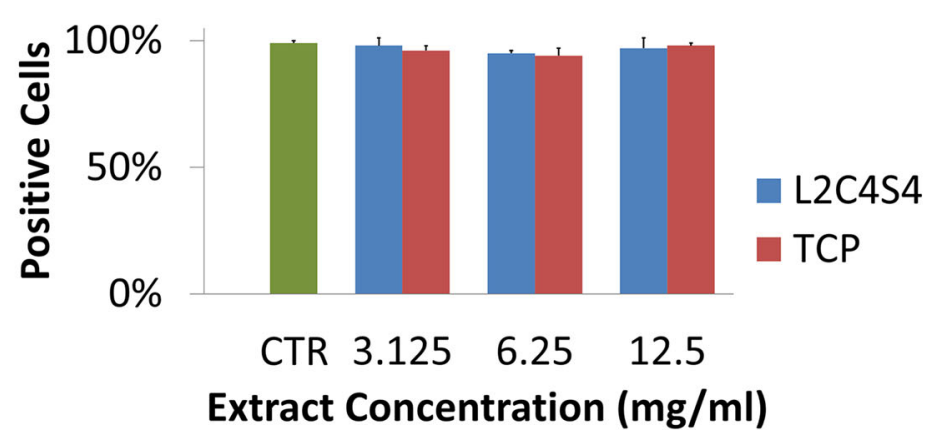

Fig. 2 Effects of L2C4S4 extracts on proliferation of iPSCs. a Immunofluorescence of Ki67 in different groups. $\mathbf{b}$ The ratio of positive cells of Ki67. Scale bar $=200 u m$. CTR: mTeSR1 medium without extracts. Data presented as mean \pm SEM

iPSCs medium did not change the pluripotency of iPSCs.

\section{Beneficial effects of L2C4S4 on the maintaining of chondrocyte spheres}

The chondrogenic differentiation medium (MCDM) with different dilutions of L2C4S4/TCP extracts was prepared for the chondrogenic differentiation of human iPSCs. The iPSCs were dissociated and diluted to form spheres for $3 \mathrm{~h}$ and then cultured as non-adherent spheres in these medium for chondrogenic differentiation for 14 days. Chondrocyte spheres cultured in MCDM without extracts was served as a control. The timeline is shown in Fig. 4a. Each chondrocyte sphere was photographed with a microscope. Similar to control and TCP, altered concentration $(3.125 \sim 12.5 \mathrm{mg} / \mathrm{mL})$ of L2C4S4 extracts promoted the formation of well-formed spheres (Fig. 4b), which indicated that the L2C4S4 could help maintain normal architecture morphologically of iPSCs-derived chondrocyte spheres.

Volumes of chondrocyte spheres were measured through Image-Pro Plus 6.0 software. To calculate the relative volume of chondrocyte spheres, the value of the control group was set as 1 . The volume of the sphere in each group was corrected according to the control group. As showed in Fig. 4c, the volumes of iPSCs-derived chondrocyte spheres in L2C4S4-treated cells were comparable to the control and TCP-treated cells. There was also no statistical difference between each group $(p>0.05)$. It demonstrated that L2C4S4 did not 


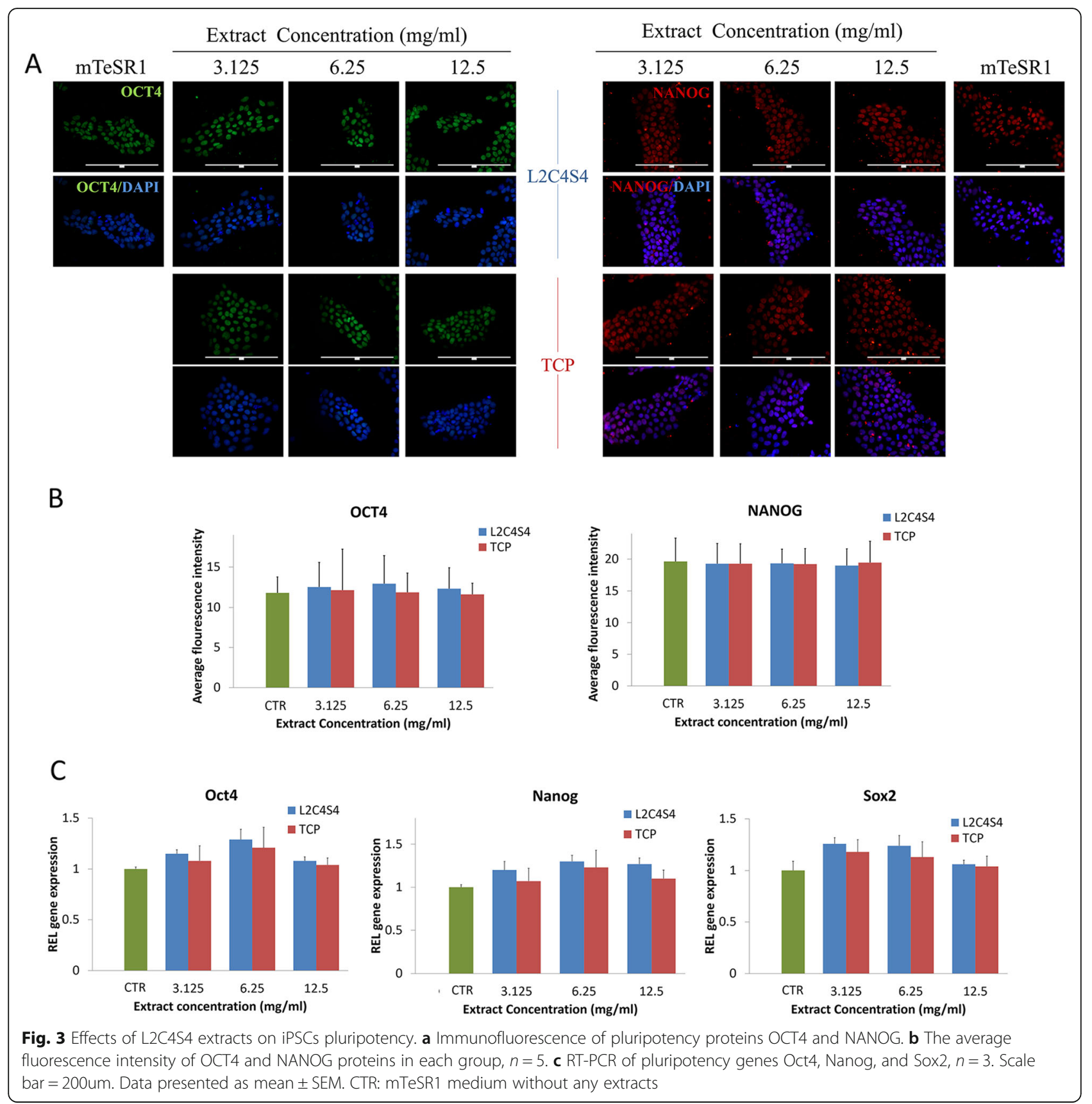

impact the chondrocyte spheres formation in an appropriate size.

The conducive stimulatory effects of L2C4S4 on chondrogenic differentiation of iPSCs

The iPSCs were cultured in MCDM with different dilutions $(3.125 \sim 12.5 \mathrm{mg} / \mathrm{mL})$ of L2C4S4 or TCP extracts for 14 days. Expression of chondrocytes specified proteins COL II, Aggrecan, and SOX9 were evaluated by immunofluorescence in chondrocyte spheres. Immunofluorescence findings showed abundant COL II, Aggrecan, and SOX9 massively gathered in the intercellular and intracellular areas of L2C4S4-treated cells while TCPtreated cells and the control group showed a small amount of these proteins' expression (Fig. 5a). The average fluorescence intensity in each group was analyzed by software Image-Pro Plus 6.0 software. Levels of COL II, Aggrecan, and SOX9 proteins in L2C4S4treated cells were more than twice as high as the other two groups (Fig. 5b, $p<0.01$ ). 


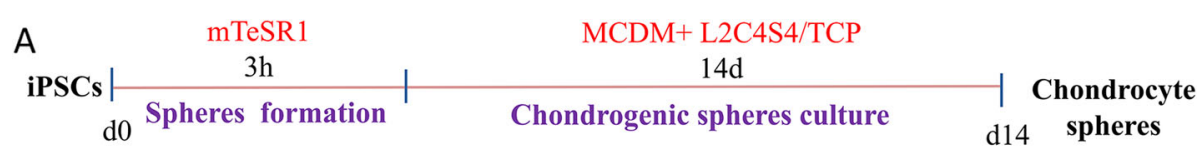

B

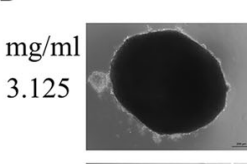

$\mathrm{L} 2 \mathrm{C} 4 \mathrm{~S} 4$
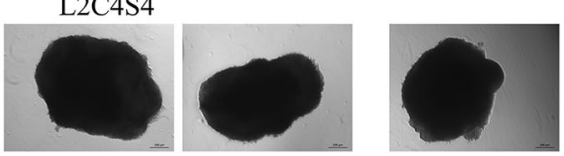

TCP

6.25
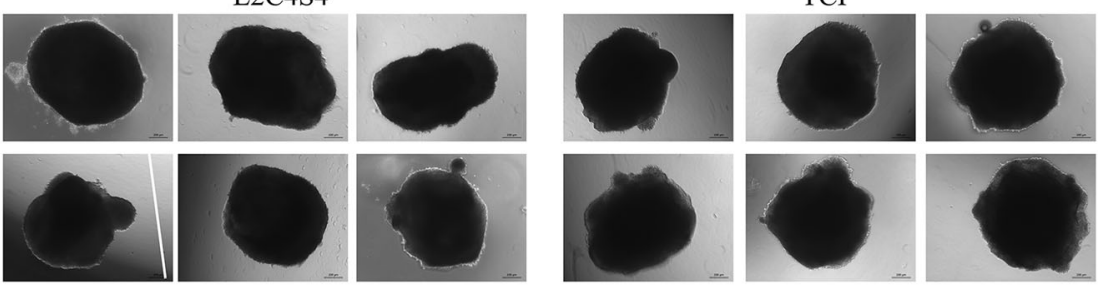

12.5
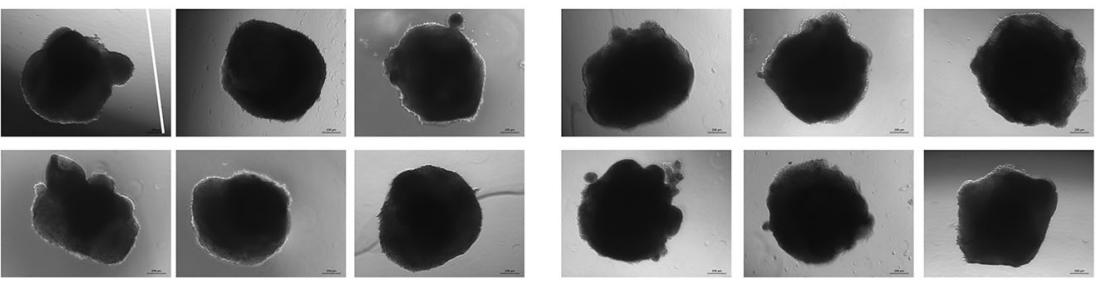

CTR
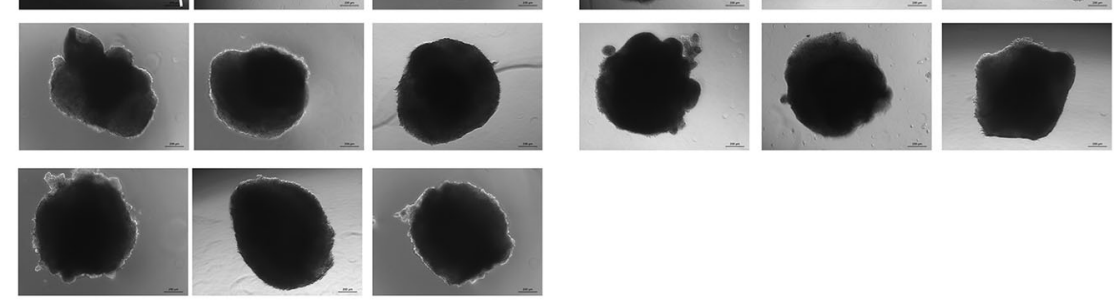

C

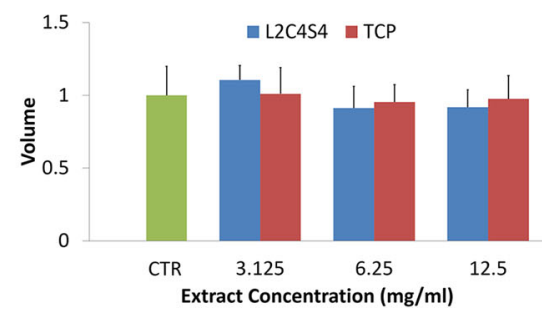

Fig. 4 Effects of L2C4S4 extracts on maintaining chondrocyte spheres. a Chondrogenic induction strategy. $\mathbf{b}$ The morphology of chondrocyte spheres in different groups, $n=3$. $\mathbf{c}$ The volume of chondrocyte spheres in different groups, $n=3$, data presented as mean \pm SEM. Scale bar $=$ $200 \mu \mathrm{m}$. CTR: MCDM without any extracts

Chondrogenic gene expression was also evaluated by RT-PCR on day 14 of chondro-induction. Expression of Col2a1, Aggrecan, and Sox9 genes in L2C4S4-treated cells was significantly enhanced at the concentration range of $3.125 \sim 12.5 \mathrm{mg} / \mathrm{mL}(p<0.05)$ as compared to control group (Fig. 5c). As compared to TCP-treated cells, the Col2a1 gene was significantly enhanced by L2C4S4 extracts at the concentration of $3.125 \mathrm{mg} / \mathrm{mL}$ $(p<0.001)$ and $12.5 \mathrm{mg} / \mathrm{mL}(p<0.01)$, and the expression of Aggrecan and Sox9 genes distinctly increased at the concentration of $3.125 \mathrm{mg} / \mathrm{mL}$ and $6.25 \mathrm{mg} / \mathrm{mL}$ $(p<0.05)$. These results indicated that the L2C4S4 had conducive stimulatory effects on the chondrogenic differentiation of iPSCs.

\section{Low level of hypertrophy in the iPSC-chondrogenic spheres cultured in extracts of L2C4S4}

In order to better determine the differentiation status of chondrocytes, chondrocyte hypertrophy markers COL X and MMP13 were analyzed after 14 days of iPSCs-chondrogenic differentiation. The results from immunofluorescence showed an obvious reduction of COL X and MMP13 in L2C4S4-treated cells than in TCP-treated cells and control group at different concentrations of extracts $(3.125 \sim 12.5 \mathrm{mg} / \mathrm{mL}, p<0.01$, Fig. 6a). The average fluorescence intensity of COL $X$ and MMP13 proteins in the control group and TCPtreated cells was more than twice as high as the L24S4-treated cells (Fig. 6b, $p<0.01$ ). Hypertrophicrelated genes COL X, Mmp13 and Indian hedgehog signaling molecule (Ihh) were also evaluated by RTPCR in each group. TCP-treated cells and control group had greater expression of these three genes than L2C4S4-treated cells $(p<0.05$, Fig. 6c). Thus, extracts of L2C4S4 could effectively reduce the level of hypertrophy in the iPSC-chondrogenic spheres.

\section{The accelerated effect of $\mathrm{Li}^{+}$ions on the chondrogenic differentiation of iPSCs}

In order to seek out which ingredient of L2C4S4 that played a crucial part in chondrogenic differentiation, the ionic concentrations of calcium (Ca), lithium ( $\mathrm{Li})$, and 


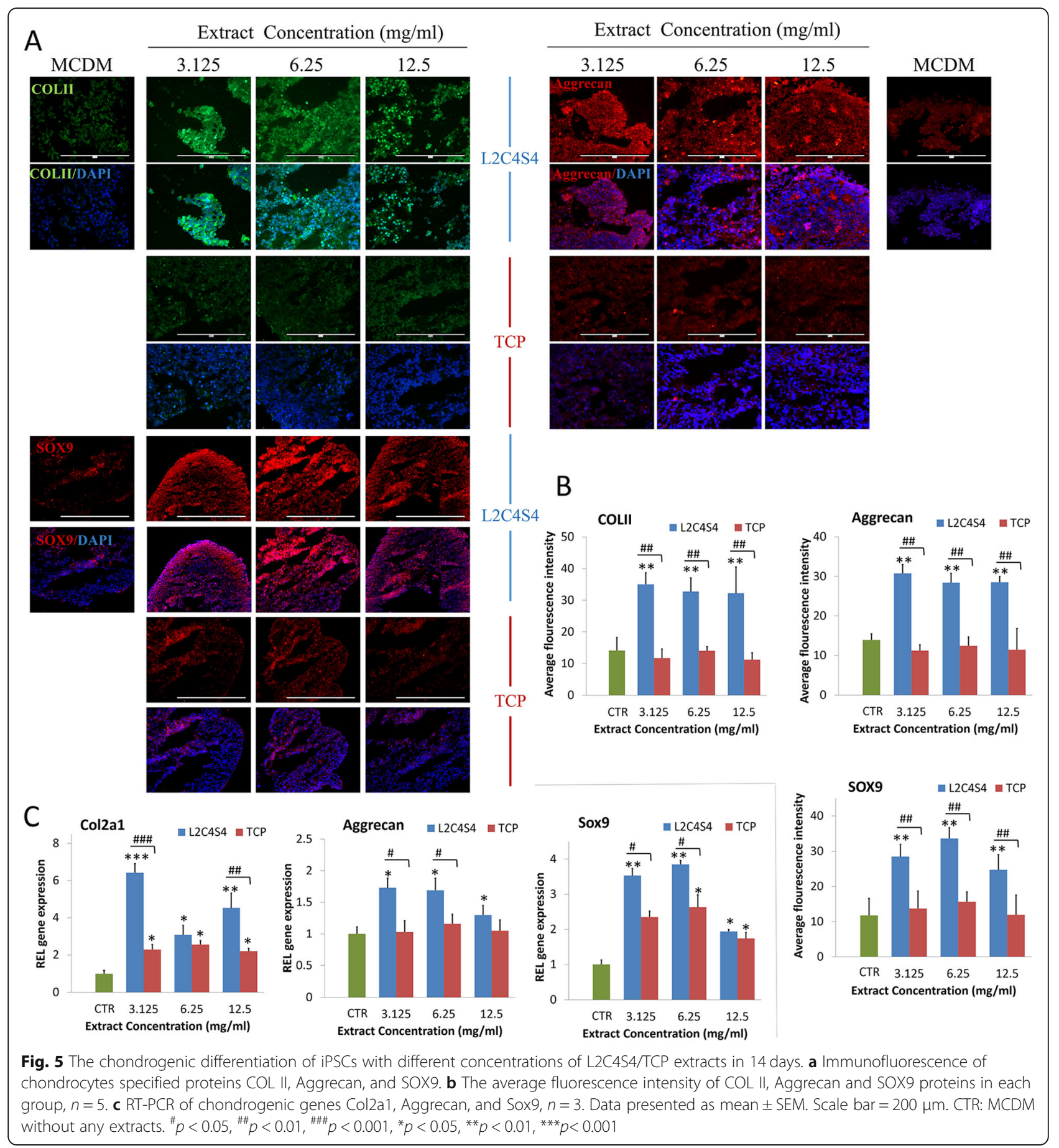

phosphorus (P) ions in graded extracts of L2C4S4 and TCP were calculated by inductively coupled plasma atomic emission spectrometry. The values were given in Table 1 . There were no significant differences between $\mathrm{Ca}$ and $\mathrm{P}$ ions in the extracts of L2C4S4 and TCP. In contrast, the $\mathrm{Li}^{+}$ions concentration was positively correlated with the concentration of the L2C4S4 extracts. Corresponding to the concentration gradient of different L2C4S4 extracts (3.125 12.5 $\mathrm{mg} / \mathrm{mL}$ ), the concentration of $\mathrm{Li}^{+}$ions varies within $5.78 \sim 23.73 \mathrm{mg} / \mathrm{L}$. In addition, there were no $\mathrm{Li}^{+}$ions existing in TCP extracts and MCDM. Based on these data, we decided to verify the effects of $\mathrm{Li}^{+}$ions on chondrogenic differentiation of iPSCs. To mimic $\mathrm{Li}^{+}$ions concentrations in $\mathrm{L} 2 \mathrm{C} 4 \mathrm{~S} 4$ extracts, $5.78 \sim 23.73 \mathrm{mg} / \mathrm{L} \mathrm{Li}^{+}$ions were prepared of $\mathrm{LiCl}$ and applied in MCDM to culture iPSCs. MCDM without any extracts was served as a control. In 


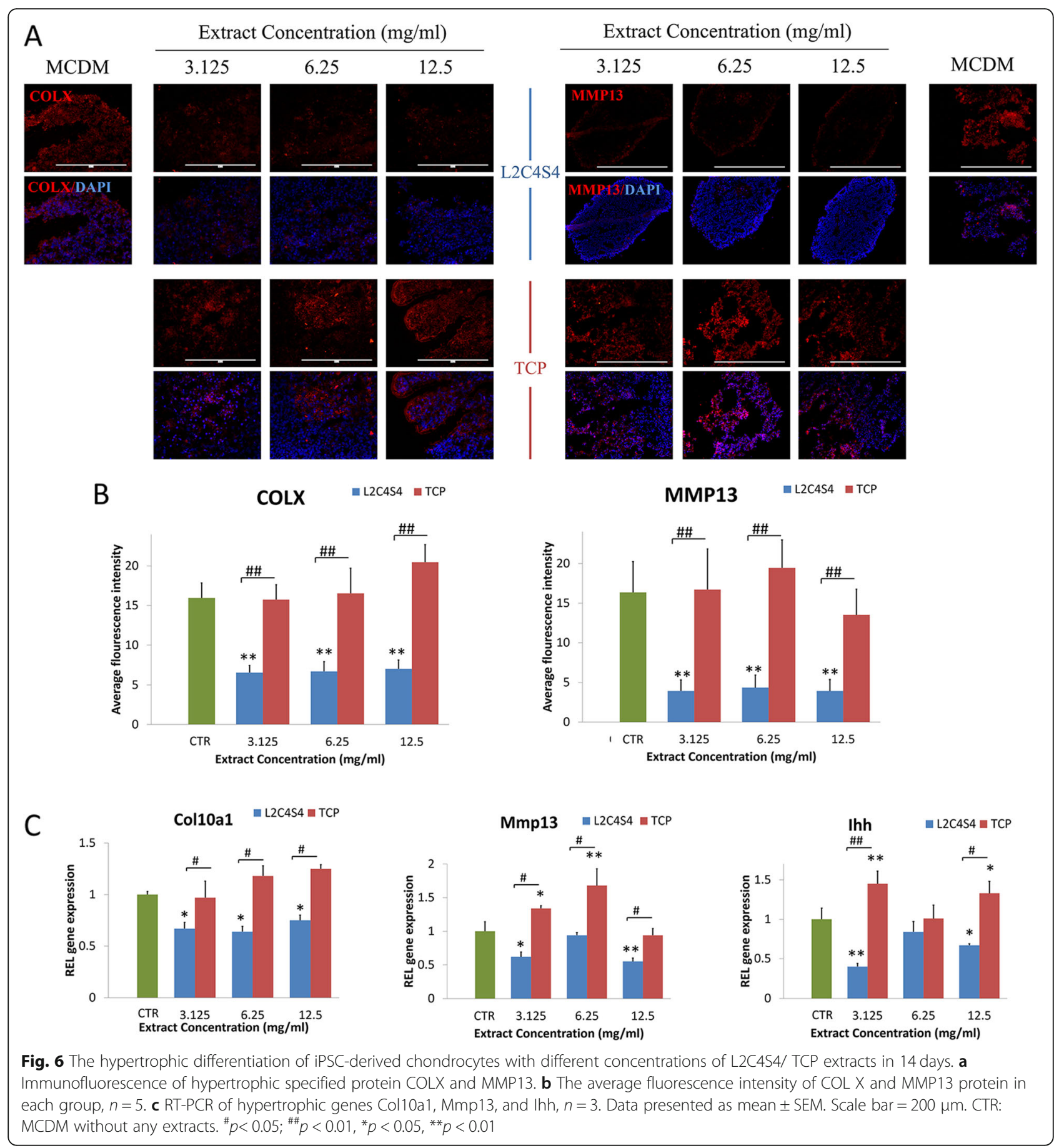

immunofluorescence analysis, the fluorescence intensity of chondrocyte proteins COL II, Aggrecan, and SOX9 in $\mathrm{Li}^{+}$ ions-treated cells was all stronger than MCDM-treated cells apparently; meanwhile, the expression of hypertrophic marker COL X and MMP13 showed the opposite trend (Fig. 7a).

The average fluorescence intensity of COL II, Aggrecan, and SOX9 proteins in $\mathrm{Li}^{+}$ions-treated cells was more than twice as high as the control group (Fig. 7b, $p<0.05$ ). The expression of chondrogenic genes Col2a1, Aggrecan, and Sox9 all rose in different concentrations of $\mathrm{Li}^{+}$ions-treated cells compared with the control group $(p<0.05$, Fig. $7 c)$. On the contrary, the expression of the Col10a1 gene in $\mathrm{Li}^{+}$ionstreated cells at the concentration range of 5.78 23.73 $\mathrm{mg} / \mathrm{L}$ was far less than the control group $(p<0.05$, 
Table 1 The ionic concentrations of $\mathrm{Ca}$, $\mathrm{Li}$ and $\mathrm{P}$ ions in graded extracts

\begin{tabular}{llll}
\hline Medium & \multicolumn{3}{l}{ Iron concentration } \\
\cline { 2 - 4 } & Ca $(\mathrm{mg} / \mathrm{L})$ & $\mathrm{Li}(\mathrm{mg} / \mathrm{L})$ & $\mathrm{P}(\mathrm{mg} / \mathrm{L})$ \\
\hline MCDM & 85.24 & - & 17.68 \\
L2C4S4 + MCDM $(3.125 \mathrm{mg} / \mathrm{mL})$ & 84.32 & 5.78 & 17.42 \\
L2C4S4 + MCDM $(6.26 \mathrm{mg} / \mathrm{mL})$ & 83.57 & 11.82 & 17.12 \\
L2C4S4 + MCDM $(12.5 \mathrm{mg} / \mathrm{mL})$ & 82.34 & 23.73 & 16.52 \\
TCP + MCDM $(3.125 \mathrm{mg} / \mathrm{mL})$ & 85.14 & - & 18.17 \\
TCP + MCDM $(6.26 \mathrm{mg} / \mathrm{mL})$ & 84.34 & - & 18.49 \\
TCP + MCDM $(12.5 \mathrm{mg} / \mathrm{mL})$ & 82.46 & - & 19.03 \\
\hline
\end{tabular}

MCDM serum-free chondrogenic differentiation medium

Fig. 7d). The Ihh gene was significantly reduced by $\mathrm{Li}^{+}$ions at the concentration of $5.78 \mathrm{mg} / \mathrm{L} \quad(p<0.05)$ and the expression of the Mmp13 gene distinctly decreased at the concentration of $11.82 \mathrm{mg} / \mathrm{L}$ and $23.73 \mathrm{mg} / \mathrm{L} \mathrm{Li}^{+}$ions $(p<0.05$, Fig. $7 \mathrm{~d})$. These data confirmed that individual $\mathrm{Li}^{+}$ions at different concentrations $(5.78 \sim 23.73 \mathrm{mg} / \mathrm{L})$ also accelerated the chondrogenic differentiation of iPSCs and significantly prevented their hypertrophy.

\section{Discussion}

In previous studies, a variety of biomaterials exhibited good effects on the maintenance of cartilage [11-15]. However, whether a biomaterial can observably promote chondrogenic differentiation of human iPSCs in vitro has not reached a firm conclusion. The toxic effect of biomaterials on iPSCs is our first consideration. Owing to the good affinity of the L2C4S4 for rabbit MSCs in our previous studies, we also tried to apply same dilutions of extracts $(3.125 \sim 12.5 \mathrm{mg} / \mathrm{mL})$ of $\mathrm{L} 2 \mathrm{C} 4 \mathrm{~S} 4$ into iPSCs culture and observed vigorous growth cells with high pluripotency, which was of great significance for the subsequent differentiation process.

Well-formed chondrocyte spheres are of profound significance for cartilage defect repair [16]. Although the technology for applying biomaterials and iPSCs to cartilage tissue engineering was becoming increasingly sophisticated [17, 18], the shape and morphology of iPSCsderived chondrocyte spheres were often overlooked. According to our results, it indicated that the use of L2C4S4 extracts can help maintain the good shape and suitable volume of iPSCs-derived chondrocyte spheres, which were roughly equivalent to spheres obtained by other induction methods $[19,20]$.

COL II and Aggrecan are key chondrocyte proteins enriched in the cartilage matrix and withstand compression in cartilage. Sox 9 is the master transcription factor of chondrogenesis and acts during chondrocyte differentiation. The substantial synthesis of these three markers meant that the iPSCs could be induced into mature chondrocytes with the help of L2C4S4 extracts. Although iPSCs could be induced into chondrocytes in various ways [6,21, 22], it still existed a little regret of time-consuming in these methods. However, our results revealed a vital function of L2C4S4 in promoting the rapid conversion of iPSCs to mature chondrocytes within only 14 days. It was a one-step differentiation method and quicker than common approaches (Fig. 8). Regardless of the fact that the induction time was greatly shortened with the help of L2C4S4 extracts, the SOX9, and Aggrecan synthesized by the new-derived chondrocytes are comparable to those from other induction methods $[23,24]$.

Articular chondrocyte prehypertrophic differentiation is essential for osteoarthritis initiation and progression [25]. Hypertrophic chondrocytes may die or survive the cartilage-to-bone transition and become osteogenic cells in endochondral bones [26]. Avoiding cartilage hypertrophy is a key challenge when repairing articular cartilage. However, the evaluation of cartilage hypertrophy was often ignored in cartilage tissue engineering [2729]. COL $X$ is a short-chain collagen expressed by hypertrophic chondrocytes during endochondral ossification and often used as a classic marker of hypertrophic chondrocytes. Mmp13 is a member of matrix metalloproteinases family and involves in the breakdown of the extracellular matrix in cartilage. Our L2C4S4 biomaterial demonstrated superior hypertrophy inhibition in iPSCsderived chondrocytes as to lower COL X and MMP13 expression. Ihh is a member of the hedgehog family and plays a role in bone growth and differentiation. This gene is also considered as important markers in hypertrophic chondrocytes. L2C4S4 extracts significantly reduced its expression in chondrocytes which indicated a lower level of hypertrophy in the iPSC-chondrogenic spheres. It revealed that these spheres were more suitable for repairing articular cartilage defects.

The most significant difference of ionic composition between $\mathrm{L} 2 \mathrm{C} 4 \mathrm{~S} 4$ extracts and MCDM was $\mathrm{Li}^{+}$ions concentration. $\mathrm{Li}^{+}$ions were proved to have significantly admirable effects on cartilage [30], such as enhancing glycosaminoglycan-rich cartilage matrix production and protecting cartilage from degradation [31, 32]. However, the role of $\mathrm{Li}^{+}$ions on iPSCs has been rarely discussed. Compared to these studies, our results further showed that $\mathrm{Li}^{+}$ions could promote iPSCs to rapid chondrogenic differentiation and inhibit their hypertrophy. It was most likely that $\mathrm{Li}^{+}$ions in L2C4S4 played the principal role. As previous studies have shown that $\mathrm{Li}^{-}$ releasing biomaterials could induce chondrogenic differentiation, hyaline cartilaginous matrix formation [33] 


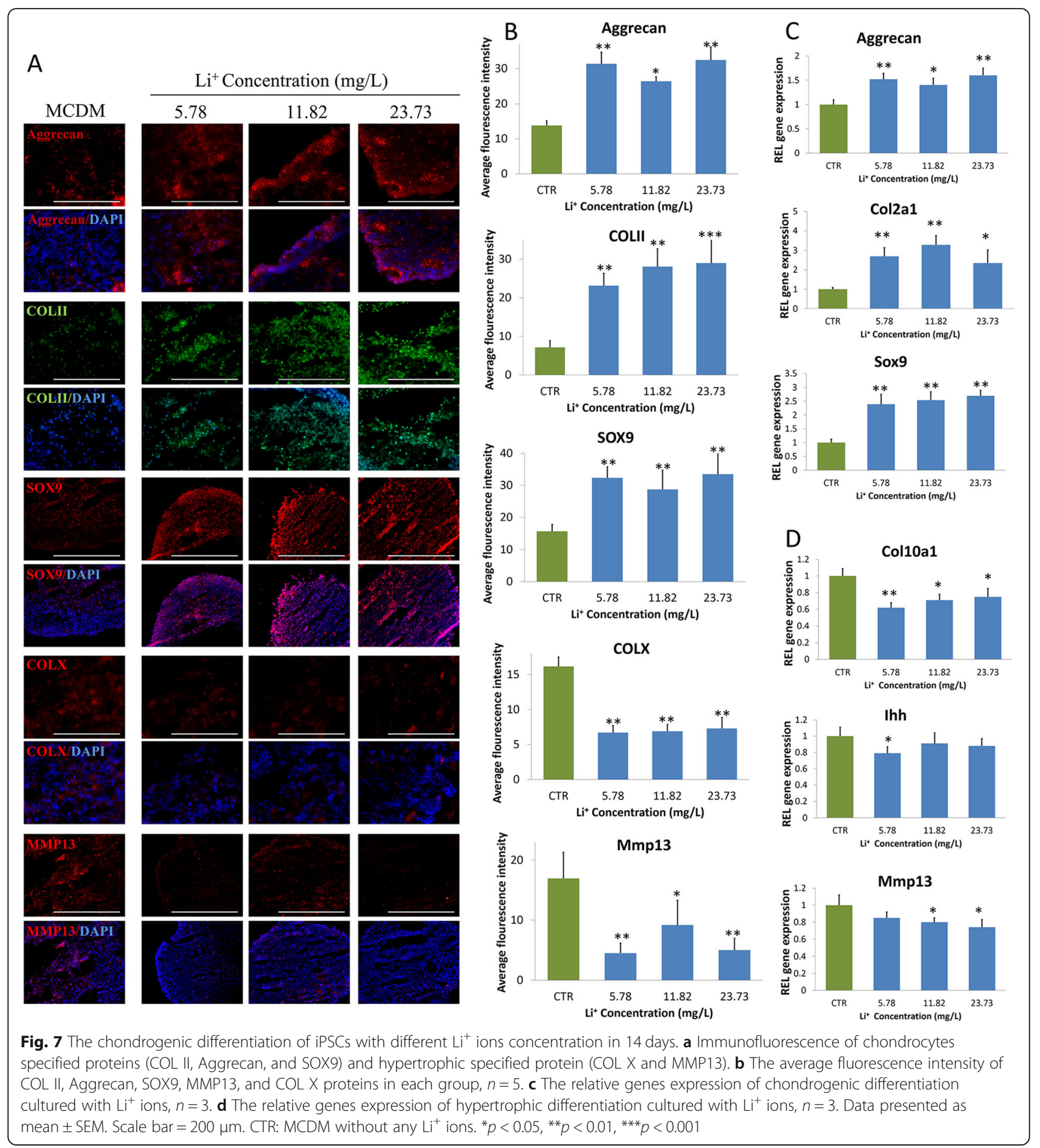

and enhance cartilage regeneration [34], our L2C4S4 bioceramics is also expected to be applicable to clinical cartilage repair due to the good release capacity of $\mathrm{Li}^{+}$ ions.

\section{Conclusions}

It is the first attempt to apply L2C4S4 bioceramics into chondrogenic differentiation of human iPSCs with a distinct function. In our study, the introduction of L2C4S4 bioceramic not only promoted the expression of COL II, Aggrecan, and SOX9 but also reduced COL X/MMP13 production in iPSC-derived chondrocytes. The L2C4S4 extracts at the concentration range of $3.25 \sim 12.5 \mathrm{mg} / \mathrm{mL}$ distinctly stimulated the fast chondrocyte differentiation of iPSCs and inhibited their final hypertrophy. Furthermore, the 


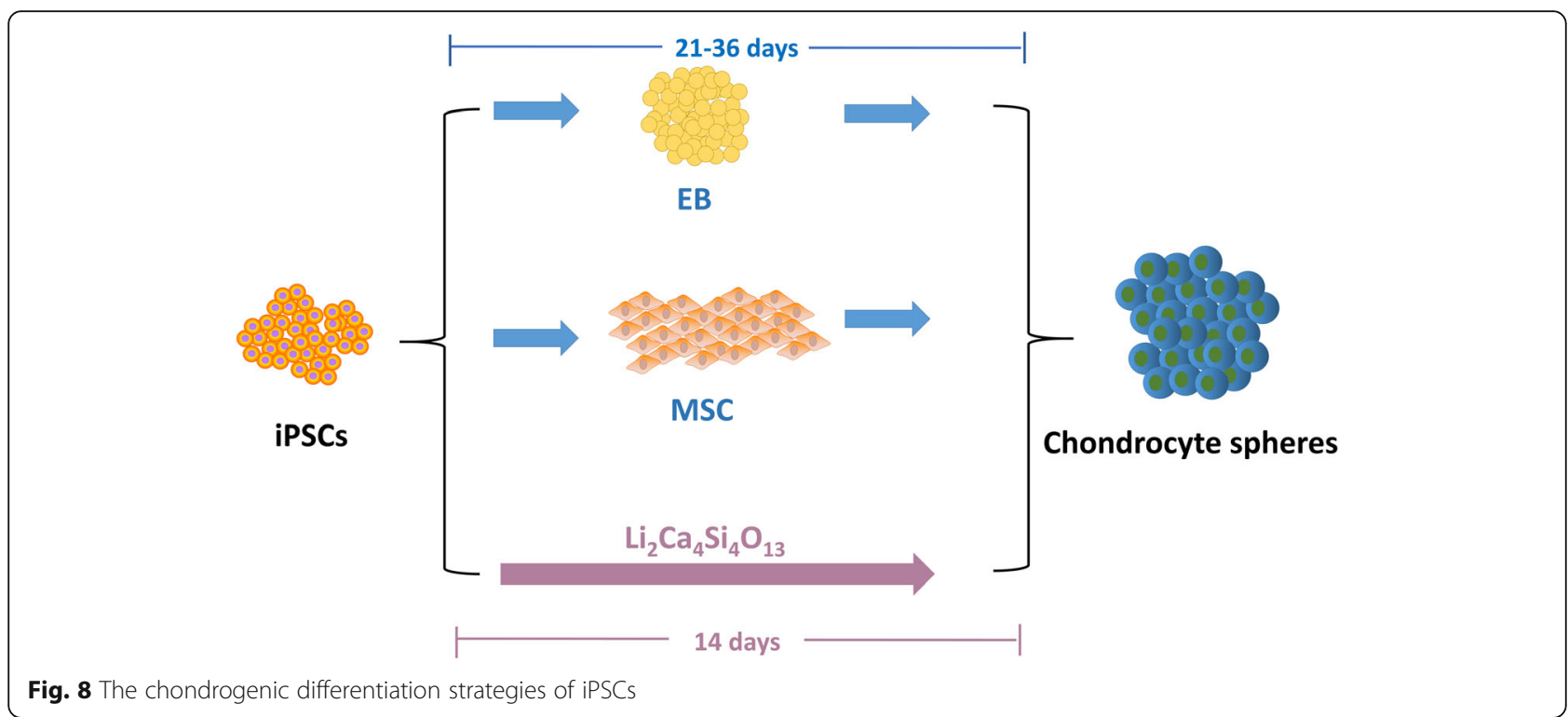

stimulatory effects of $\mathrm{Li}^{+}$ions in chondrogenic differentiation of iPSCs showed similar results, which proved that $\mathrm{Li}^{+}$ions in $\mathrm{L} 2 \mathrm{C} 4 \mathrm{~S} 4$ extracts may play a major role in the differentiation process. These results revealed that $\mathrm{L} 2 \mathrm{C} 4 \mathrm{~S} 4$ bioceramic can biologically meet the demands of rapid chondrogenesis of human iPSCs and may serve as a useful platform for cartilage repair in the future. This study offered a viable new strategy for articular cartilage regeneration from an adequate source.

\section{Supplementary information}

Supplementary information accompanies this paper at https://doi.org/10. 1186/s13287-020-01606-w.

Additional file 1:. Table S1. Designations, sequences, and the sizes of RT- PCR amplicons.

\section{Abbreviations}

iPSCs: Induced pluripotent stem cells; L2C4S4: Lithium-containing bioceramic; COL II: Type II collagen; COL X: Type X collagen; MSCs: Mesenchymal stem cells; EBs: embryoid bodies; Mmp13: Matrix metallopeptidase 13; SOX9: SRYBox 9; Ihh: Indian hedgehog signaling molecule; Ca: Calcium; Li: Lithium; P: Phosphorus; RT-PCR: Real-time quantitative polymerase chain reaction; TCP: Tricalcium phosphate

\section{Acknowledgements}

Not applicable.

\section{Authors' contributions}

YH and LY designed the research. YH and LC performed the research. PC and $Y G$ analyzed the data. YH and CW wrote the paper. LY and QJ conceived of the study, reviewed the data, and approved the final version of the manuscript. All authors read and approved the final manuscript.

\section{Funding}

This work was supported by the National Key Research and Development Program of China (2018YFC1105201), National Natural Science Foundation of China $(81472043,81871743)$, Natural Science Foundation of Shaanxi Province (2017ZDJC-12), and Shanghai Nature Science Foundation(19ZR1464800).
Availability of data and materials

All supporting data are included in the article and its additional files.

Ethics approval and consent to participate

All procedures performed in studies involving human tissue were in accordance with the ethical standards of the institutional and/or national research committee and with the 1964 Helsinki Declaration and its later amendments or comparable ethical standards. Trials were approved by the Ethics of First Affiliated Hospital of Fourth Military Medical University (permission code XJYYLL-2014076).

\section{Consent for publication}

Not applicable.

\section{Competing interests}

The authors declare that they have no competing interests.

\section{Author details}

'Department of Orthopedic Surgery, Honghui Hospital, College of Medicine, Xi'an Jiaotong University, Xi'an 710049, People's Republic of China. ${ }^{2}$ Institute of Orthopedic Surgery, Xijing Hospital, Fourth Military Medical University, Xi'an 710032, People's Republic of China. ${ }^{3}$ State Key Laboratory of High Performance Ceramics and Superfine Microstructure, Shanghai Institute of Ceramics, Chinese Academy of Science, Shanghai 200050, People's Republic of China.

Received: 8 December 2019 Revised: 10 February 2020

Accepted: 14 February 2020 Published online: 21 February 2020

\section{References}

1. Nam Y, Rim YA, Jung SM, Ju JH. Cord blood cell-derived iPSCS as a new candidate for chondrogenic differentiation and cartilage regeneration. Stem Cell Res Ther. 2017;8:16.

2. Nguyen D, Hagg DA, Forsman A, Ekholm J, Nimkingratana P, Brantsing C, et al. Cartilage tissue engineering by the 3D bioprinting of iPS cells in a Nanocellulose/alginate bioink. Sci Rep. 2017;7:658.

3. Lee J, Taylor SEB, Smeriglio P, Lai J, Maloney WJ, Yang F, et al. Early induction of a prechondrogenic population allows efficient generation of stable chondrocytes from human induced pluripotent stem cells. FASEB J. 2015;29:3399-410.

4. Lietman SA. Induced pluripotent stem cells in cartilage repair. World J Orthopedics. 2016;7:149.

5. Guzzo RM, Gibson J, Xu R, Lee FY, Drissi H. Efficient differentiation of human iPSC-derived mesenchymal stem cells to chondroprogenitor cells. J Cell Biochem. 2013;114:480-90 
6. Yamashita A, Morioka M, Yahara Y, Okada M, Kobayashi T, Kuriyama S, et al. Generation of Scaffoldless hyaline cartilaginous tissue from human iPSCs. Stem Cell Rep. 2015:4:404-18.

7. Nasu A, lkeya M, Yamamoto T, Watanabe A, Jin Y, Matsumoto Y, et al. Genetically matched human iPS cells reveal that propensity for cartilage and bone differentiation differs with clones, not cell type of origin. PLoS One. 2013;8:e53771.

8. Liu J, Nie H, Xu Z, Niu X, Guo S, Yin J, et al. The effect of 3D nanofibrous scaffolds on the chondrogenesis of induced pluripotent stem cells and their application in restoration of cartilage defects. PLoS One. 2014;9:e111566.

9. He P, Fu J, Wang DA. Murine pluripotent stem cells derived scaffold-free cartilage grafts from a micro-cavitary hydrogel platform. Acta Biomater. 2016;35:87-97.

10. Chen L, Deng C, Li J, Yao Q, Chang J, Wang L, et al. 3D printing of a lithium-calcium-silicate crystal bioscaffold with dual bioactivities for osteochondral interface reconstruction. Biomaterials. 2019;196:138-50.

11. Shao X, Lin S, Peng Q, Shi S, Wei X, Zhang T, et al. Tetrahedral DNA nanostructure: a potential promoter for cartilage tissue regeneration via regulating chondrocyte phenotype and proliferation. Small. 2017;13:1602770.

12. Mhanna R, Kashyap A, Palazzolo G, Vallmajo-Martin Q, Becher J, Möller S, et al. Chondrocyte culture in three dimensional alginate sulfate hydrogels promotes proliferation while maintaining expression of chondrogenic markers. Tissue Eng A. 2014;20:1454-64.

13. Kim YS, Park DY, Cho YH, Chang JW, Choi JW, Park JK, et al. Cultured chondrocyte and porcine cartilage-derived substance (PCS) construct as a possible dorsal augmentation material in rhinoplasty: a preliminary animal study. J Plast Reconstr Aesthet Surg. 2015;68:659-66.

14. Yang X, Lu Z, Wu H, Li W, Zheng L, Zhao J. Collagen-alginate as bioink for three-dimensional (3D) cell printing based cartilage tissue engineering. Mater Sci Eng C Mater Biol Appl. 2018;83:195-201.

15. Joergensen NL, Le DQS, Andersen OZ, Foss M, Danielsen CC, Foldager CB, et al. Topography-guided proliferation: distinct surface microtopography increases proliferation of chondrocytesIn vitro. Tissue Eng A. 2015;21:2757-65.

16. Yin L, Wu Y, Yang Z, Denslin V, Ren X, Tee CA, et al. Characterization and application of size-sorted zonal chondrocytes for articular cartilage regeneration. Biomaterials. 2018;165:66-78.

17. Imaizumi M, Nomoto $Y$, Sato $Y$, Sugino T, Miyake $M$, Wada I, et al. Evaluation of the use of induced pluripotent stem cells (iPSCs) for the regeneration of tracheal cartilage. Cell Transplant. 2013;22:341-53.

18. Diekman BO, Christoforou N, Willard VP, Sun H, Sanchez-Adams J, Leong KW, et al. Cartilage tissue engineering using differentiated and purified induced pluripotent stem cells. Proc Natl Acad Sci U S A. 2012;109:19172-7.

19. Saito T, Yano F, Mori D, Ohba S, Hojo H, Otsu M, et al. Generation of Col2a1EGFP iPS cells for monitoring chondrogenic differentiation. PLoS One. 2013; 8:e74137.

20. Diederichs S, Gabler J, Autenrieth J, Kynast KL, Merle C, Walles H, et al. Differential regulation of SOX9 protein during chondrogenesis of induced pluripotent stem cells versus Mesenchymal stromal cells: a shortcoming for cartilage formation. Stem Cells Dev. 2016;25:598-609.

21. Driessen B, Logie C, Vonk LA. Cellular reprogramming for clinical cartilage repair. Cell Biol Toxicol. 2017;33:329-49.

22. Yamashita A, Morioka M, Kishi H, Kimura T, Yahara Y, Okada M, et al. Statin treatment rescues FGFR3 skeletal dysplasia phenotypes. Nature. 2014;513:507-11.

23. Lach MS, Wroblewska J, Kulcenty K, Richter M, Trzeciak T, Suchorska WM. Chondrogenic differentiation of pluripotent stem cells under controllable serum-free conditions. Int J Mol Sci. 2019;20:2711.

24. Xu M, Stattin E, Shaw G, Heinegård D, Sullivan G, Wilmut I, et al. Chondrocytes derived from Mesenchymal stromal cells and induced pluripotent cells of patients with familial Osteochondritis Dissecans exhibit an endoplasmic reticulum stress response and defective matrix assembly. Stem Cell Transl Med. 2016;5:1171-81.

25. Zhang $\mathrm{H}$, Wang $\mathrm{H}$, Zeng $\mathrm{C}$, Yan B, Ouyang J, Liu $\mathrm{X}$, et al. mTORC1 activation downregulates FGFR3 and PTH/PTHrP receptor in articular chondrocytes to initiate osteoarthritis. Osteoarthr Cartil. 2017;25:952-63.

26. Yang L, Tsang KY, Tang HC, Chan D, Cheah KS. Hypertrophic chondrocytes can become osteoblasts and osteocytes in endochondral bone formation. Proc Natl Acad Sci U S A. 2014;111:12097-102.

27. Tsumaki N, Okada M, Yamashita A. iPS cell technologies and cartilage regeneration. Bone. 2015;70:48-54.

28. Xu X, Shi D, Liu Y, Yao Y, Dai J, Xu Z, et al. In vivo repair of full-thickness cartilage defect with human iPSC-derived mesenchymal progenitor cells in a rabbit model. Exp Ther Med. 2017;14:239-45.
29. Zhu Y, Wu X, Liang Y, Gu H, Song K, Zou X, et al. Repair of cartilage defects in osteoarthritis rats with induced pluripotent stem cell derived chondrocytes. Bmc Biotechnol. 2016;16:78-88.

30. Minashima T, Zhang $Y$, Lee $Y$, Kirsch T. Lithium protects against cartilage degradation in osteoarthritis. Arthritis Rheumatol. 2014;66:1228-36.

31. Hui W, Litherland GJ, Jefferson M, Barter MJ, Elias MS, Cawston TE, et al. Lithium protects cartilage from cytokine-mediated degradation by reducing collagen-degrading MMP production via inhibition of the P38 mitogenactivated protein kinase pathway. Rheumatology. 2010;49:2043-53.

32. Thompson CL, Yasmin H, Varone A, Wiles A, Poole CA, Knight MM. Lithium chloride prevents interleukin-1 $\beta$ induced cartilage degradation and loss of mechanical properties. J Orthop Res. 2015;33:1552-9.

33. Li S, Macon A, Jacquemin M, Stevens MM, Jones JR. Sol-gel derived lithiumreleasing glass for cartilage regeneration. J Biomater Appl. 2017:32:104-13.

34. Li J, Yao Q, Xu Y, Zhang H, Li L, Wang L. Lithium chloride-releasing 3D printed scaffold for enhanced cartilage regeneration. Med Sci Monitor. 2019; 25:4041-50

\section{Publisher's Note}

Springer Nature remains neutral with regard to jurisdictional claims in published maps and institutional affiliations.

Ready to submit your research? Choose BMC and benefit from:

- fast, convenient online submission

- thorough peer review by experienced researchers in your field

- rapid publication on acceptance

- support for research data, including large and complex data types

- gold Open Access which fosters wider collaboration and increased citations

- maximum visibility for your research: over $100 \mathrm{M}$ website views per year

At BMC, research is always in progress.

Learn more biomedcentral.com/submissions 